\title{
Current Concepts in the Management of the Rheumatoid Hand
}

\author{
Kevin C Chung, M.D., M.S. ${ }^{1}$ and Allison G Pushman, BA ${ }^{2}$ \\ ${ }^{1}$ Professor of Surgery, Section of Plastic Surgery, Department of Surgery, The University of \\ Michigan Health System \\ ${ }^{2}$ Research Assistant, Section of Plastic Surgery, Department of Surgery, The University of \\ Michigan Health System
}

\begin{abstract}
Hand surgeons are an integral part of the management team for patients with rheumatoid arthritis. There is now a greater understanding of the national utilization of rheumatoid hand surgery, which highlights the differences between hand surgeons and rheumatologists regarding the treatment of the rheumatoid hand. Advances in medical treatments have also decreased the prevalence of hand deformities caused by this disease. Hand surgeons today have less exposure to treating rheumatoid hand, but despite more effective medical options, surgery may still offer patients hope for improvement of hand function and appearance. This article will summarize the current state of rheumatoid hand surgery and discuss the surgical treatment strategies for optimizing outcomes for patients suffering from rheumatoid arthritis.
\end{abstract}

\section{Keywords}

Rheumatoid Arthritis; Review; Outcomes; Surgery; Medical Treatment

The treatment of the rheumatoid hand is shrouded in uncertainty and controversy. The disagreements between hand surgeons and rheumatologists regarding the indications, timing and effectiveness of rheumatoid hand surgery have persisted, despite recent studies that highlighted the wide rift between these two specialties in caring for this population.(1-4) With the introduction of new medications such as etanercept, infliximab, and adalimumab, which are several of the biologic medications used to treat rheumatoid arthritis (RA), the prevalence of hand deformities in the US may have decreased and hand surgeons are reporting seeing less severe deformities. However, these biologics are exceedingly expensive. A study conducted in 2003 demonstrated that since the introduction of biologics, the annual cost of treating an RA patient in the US has increased 3-fold.(5) Additionally, in our current Silicone Metacarpophalangeal Joint Arthroplasty (SMPA) Trial, we have found that biologics are used rather routinely in the US, but are rarely used in the United Kingdom (UK).(6) The cost of biologics may dissuade the National Health Service in the UK and the nations in the European Union to permit the general use of this medication class, which may explain the continued large experience in the European nations in treating rheumatoid hand problems.

(C) 2011 Published by Elsevier Inc on behalf of The American Society for Surgery of the Hand.

Corresponding Author: Kevin C. Chung, MD, MS, Section of Plastic Surgery, University of Michigan Health System, 2130 Taubman Center, 1500 E. Medical Center Drive, Ann Arbor, MI, 48109-0340, kecchung@umich.edu, Phone 734-936-5885, Fax 734-763-5354.

Publisher's Disclaimer: This is a PDF file of an unedited manuscript that has been accepted for publication. As a service to our customers we are providing this early version of the manuscript. The manuscript will undergo copyediting, typesetting, and review of the resulting proof before it is published in its final citable form. Please note that during the production process errors may be discovered which could affect the content, and all legal disclaimers that apply to the journal pertain. 
Rheumatoid arthritis affects $1 \%$ of the US population(7) and in a study conducted in 1998, it was estimated that over $70 \%$ of RA patients complain of hand and wrist dysfunction.(8) Although the incidence of RA hand problems is decreasing in the US due to the more effective RA medications in preventing synovitis, it is still quite possible that many rheumatoid patients who are refractory to these medications are still not being referred for hand surgery consultation. Rheumatologists, in general, consider referring to hand surgery as a failure of the medical treatment rather than engaging hand surgery as a partner in the overall management of the rheumatoid patient.

Our studies have shown large area variation across the US for common surgical procedures for the RA hand. $(9,10)$ (Figure 1) This finding implies an inconsistency in delivery of surgical care for the rheumatoid patient with hand problems. Subsequent national surveys of opinions of rheumatologists and hand surgeons revealed a stark disagreement between these two specialties regarding the indications, effectiveness, and outcomes of rheumatoid hand surgery.(2) One of the main reasons for the persistent disagreement is that the level of evidence is low in supporting the effectiveness of RA hand surgery.(11) For this reason, outcomes research is critical in assessing the effectiveness of various RA hand surgery procedures in determining whether these procedures are effective in improving the overall health-related quality of life for patients with this debilitating disease. The purpose of this article is to highlight the recent advances in the treatment of RA and update the innovations in the treatment of the RA hand and wrist. This paper will try to provide a practical guide in the sequential and coordinated approaches in evaluating and treating complex rheumatoid hand and wrist problems.

\section{Background}

Rheumatoid arthritis is a common inflammatory arthritis resulting from a $\mathrm{T}$ cell-driven autoimmune process. In individuals with RA, destructive molecules infiltrate the synovium, often resulting in synovial hypertrophy, joint destruction, and inflammation of the supporting ligaments of joints. The cause of this auto-immune disease is unknown, but it is postulated to be a multifactorial disease, with both genetic and environmental contributions.

From an environmental standpoint, RA was a relatively uncommon disease prior to the industrial revolution. However, with the introduction of potential toxins such as silica dust $(12,13)$ during the industrial revolution, there was a consistent and rapid increase in the incidence of RA and other autoimmune disorders. Additionally, exposure to carcinogens has been shown to increase a person's risk of RA, with several studies indicating smoking as a risk factor associated with the development of RA. $(14,15)$

The genetic component of susceptibility to RA has been suggested by familial aggregation and twin studies, $(16,17)$ and has been examined in some detail in various studies. From these investigations, two genes have been shown to be strongly associated with RA susceptibility, PTPN22 $(18,19)$ and HLA-DRB1.(20) A nonsynonymous single nucleotide polymorphism located in the PTPN22 gene is implicated in the pathogenesis of RA and other autoimmune disorders, (18) whereas multiple HLA-DRB1 alleles encoding for a shared epitope at amino acid positions 70-74 are associated with susceptibility and severity of RA. $(21,22)$ HLA genes are estimated to account for $37 \%$ of the genetic contribution to RA.(23)

\section{Medical Treatment of Rheumatoid Arthritis}

Treating the rheumatoid hand is an often-arduous undertaking with no one, right answer. To complicate matters, rheumatologists and hand surgeons often disagree on the best treatment for RA patients, with hand surgeons advocating for surgery and rheumatologists resisting it. In fact, many rheumatologists do not believe that surgery for RA hand deformities is 
particularly effective. $(1,2)$ Instead, rheumatologists generally prefer to treat RA hand deformities with medications.

Medical treatment for RA patients aims for the containment of chronic inflammation as well as structural protection of the affected joints.(24) There are three general classes of drugs often used in the treatment of RA: non-steroidal anti-inflammatory agents (NSAIDs), corticosteroids, and disease modifying anti-rheumatic drugs (DMARDs). NSAIDs are used to reduce acute inflammation, thereby relieving pain and improving function; however, they do not alter the course of the disease or prevent joint destruction. Corticosteroids such as prednisone and methylprenisoline are used to reduce inflammation and regulate immune system activity when NSAIDs are no longer able to control the symptoms in severely diseased patients.(25) Corticosteroids are generally very effective; however, their value is often counterbalanced by multiple adverse side-effects ranging from mild irritability to severe and life-threatening cardiovascular events and adrenal insufficiency.(26)

Although NSAIDs and corticosteroids are successful in relieving the symptoms associated with RA, they do not change the disease course or help improve radiographic outcomes. Only disease-modifying antirheumatic drugs (DMARDs) are found to have these particular salutary effects and are commonly used in the medical treatment of RA.(27) It has been shown that in order to help reduce structural damage early on, the majority of RA patients should be started on DMARDs as soon as a diagnosis of RA is confirmed.(28) DMARDs have anti-inflammatory effects along with the structural-modifying properties and can be used for ongoing, long-lasting control of RA disease activity.(24)

There are two subgroups of DMARDs: nonbiologic and biologic agents. The most common nonbiologic DMARD is methotrexate, an effective drug that can be used for several years with little toxicity. Methotrexate used to stand alone as the "gold" standard for treating RA patients; however, major advancements in the past decade for treatment of RA show that methotrexate is best used in a new role, as an anchor drug in combination with biologic agents.

Biologic agents offer safer and more effective therapeutic options but at considerably increased cost over conventional DMARDs. $(27,29)$ Two classes of biologic agents exist for RA treatment: tumor necrosis factor (TNF) inhibitors (which include etanercept, infliximab, and adalimumab) and interleukin-1 receptor antagonists (for example anakinra).(27) These agents neutralize cytokines that specifically mediate the inflammatory process that underlies RA pathogenesis. They have been shown to appreciably slow radiographic progression while improving the functional status of RA patients.(27) Most biologics act much faster than synthetic DMARDs and are accompanied by less radiological deterioration.(30) However, increasing evidence shows that combining a biologic with a conventional DMARD agent, particularly methotrexate, tends to improve RA signs and symptoms, halt radiographic progression, and induce remission even more than the use of a biologic agent alone.(27, 31-34) Etanercept, infliximab, and adalimumab are the major TNF biologic inhibitors approved for RA. Anakinra is an IL-1 receptor antagonist that can be used for RA patients who have had inadequate response to DMARD therapy.(27)

Biologics work well; however, they are quite expensive. A study conducted in 2001 found that the mean total annual direct medical care cost for a patient with RA receiving biologic therapy was $\$ 19,016$, whereas the cost for those who were not was $\$ 6,164$.(5) In order to evaluate whether these expensive biologic agents are cost-effective, the National Health System R\&D Health Technology Assessment Program (29) conducted a systematic review of the clinical effectiveness and cost-effectiveness of the TNF inhibitors adalimumab, etanercept, and infliximab for the treatment of RA adults. This report concluded that TNF 
inhibitors are most cost-effective when used as a last active therapy, and sensitive analysis showed that, with all things being equal, etanercept may be the biologic of choice; however, all are quite costly.

New medical advances have been quite effective in treating patients with RA; however, there are cases in which these medications are administered too late in the disease stage or are simply unsuccessful for particular patients. Rheumatologists and hand surgeons therefore need to work closely and coordinate care for the RA patient. Although these medications have improved outcomes and functions for the RA hand, hand surgery may still be indicated for many patients.

\section{Surgeon's Perspective}

Collaboration between surgeons and rheumatologists is paramount to the successful management of RA hand problems. Rheumatologists serve as a coordinator for the overall care of the RA patient treated by various specialists, many of whom include hand surgeons. From a hand surgeon's perspective, referral to the hand surgeon does not equate to a failure of medical management, but rather an introduction of the patient to the hand surgeon who can plan current and future care. An RA patient presenting to a hand surgery clinic does not mean an immediate surgical plan, but this initial consultation gives the patient the opportunity to participate in a shared decision-making process to arrive at a mutually agreeable treatment plan.

An RA patient with hand deformity does not necessary need reconstruction. Certain RA patients may have acclimated quite well to this disease, despite obvious hand deformities. An experienced RA hand surgeon will strive to understand the patient's needs, expectations for improvement, and the available surgical options that may predictably improve the patient's function. The surgeon must also consider the underlying disease, which may require different surgical approaches. For example, a patient with RA and lupus may present with marked ulnar deviation of the fingers without much metacarpophalangeal joint (MCP) destruction. An injudicious MCP joint arthroplasty for this patient may result in early recurrence of ulnar subluxation of the fingers at the MCP joint level if lupus is the predominant disease. This is because laxity in the ligament, not the joint destruction, is the key finding in lupus. On the other hand, a well-timed thumb MCP joint fusion can predictably increase this patient's hand function by stabilizing the thumb MCP joint.

Understanding the priority of treatment is also critical in optimizing outcome. For example, a patient presenting with MCP joint destruction and boutonniere deformity of the fingers may achieve sufficient motion following MCP joint arthroplasty that may obviate the need for PIP joint surgery. These considerations are quite important for a surgeon engaged in rheumatoid hand surgery in order to solicit the patient's input regarding his or her needs prior to planning a surgical strategy.

\section{Wrist}

The wrist is the most commonly affected joint in RA. Synovitis in the wrist joint weakens the ligamentous support of the distal radioulnar joint. Collapse of the radial column of the carpal bones results in a relative lengthening of the distal ulna in relationship with the distal radius. The typical caput ulna appearance, in which the ulna head dislocates dorsally, results in distal radioulnar joint incongruity and impaction of the distal ulna on the carpus. (Figure 2) Despite the destructive appearance of the carpus on the radiographs, many RA patients do not complain of much discomfort and are able to accommodate to their deformity. Surgical treatment is undertaken primarily to alleviate wrist pain or to correct wrist deformity that contributes to the deformities of the fingers. The progression of wrist deformity is well 
described by Taleisnik (35) who postulated the sequential, predictable events in the RA wrist. The scapho-lunate ligament is prone to weakening from the synovitis, which leads to flexion of the scaphoid and collapse of the radial column. Stretching of the wrist ulnar collateral ligament attenuates the ulnar column support. These 2 events ultimately lead to the typical carpal supination pattern. It has been observed that the carpus may sublux in an ulnar direction along the inclined radius. The consequence of carpal supination is the collapse of the radial wrist, which contributes to the radial deviation of the metacarpals and accentuates the ulnar deforming forces on the fingers at the MCP joints. The wrist joint may also sublux in an anterior direction, which causes difficulties with wrist extension.

Treatment of wrist deformities can be considered as prophylactic or corrective. An example of a prophylactic operation is the radiolunate fusion procedure. When the carpus subluxes ulnarly, it creates an imbalance in the tendon forces that contribute to the ulnar deviation of the fingers. If the midcarpal joint is relatively free of disease, the radiolunate fusion, which was described by Chamay,(36) will stabilize the wrist and allow motion through the midcarpal joint. Interestingly, studies have shown long-term maintenance of the integrity of the midcarpal joint after radiolunate fusion.(37) For DRUJ problems, there are two operations that are effective: The Darrach and the Suave-Kapandji procedures. Darrach first described resecting the distal ulna in 1912 for a patient with anterior ulna subluxation after trauma.(38) This is a time-honored procedure that is effective in alleviating pain from DRUJ disease and distal ulna impingement on the carpus. The main concern of the Darrach procedure for RA is the potential ulnar translation of the carpus for patients with weak ligamentous support. For patients whose lunates are already migrating ulnarly, a concomitant radio-lunate fusion can be performed, provided that the midcarpal joint is intact. Alternatively, the Suave-Kapandji procedure should be considered; this procedure fuses the distal radio-ulnar joint in combination with a proximal ulna ostectomy to provide rotatory function. The preserved ulna head gives support to the ulnar carpus and prevents ulnar translation of the carpus. A potential problem is the unpredictable fusion of the DRUJ when the bone stock in RA is not sufficient. An interesting variation of this procedure is the Masada's shelf arthroplasty procedure(39)(Figure 3) in which the excised distal ulna is turned 90 degrees and inserted into the radius to promote better bone contact for fusion. The turned ulna resembles a bookshelf to support the ulnar carpus. There is a recent interest in replacing the distal ulna with prosthesis. The long-term outcome of this procedure has not been proven. Dislocation and loosening of the implant are concerning problems with the ulnar head prosthesis.

For patients with debilitating pain of the radiocarpal joint, the choices are rather straightforward, with either fusion or arthroplasty procedure. Limited fusion procedures have been shown to retard the progression of deformities in the RA wrist. Most common of which is radiocarpal fusion, in which the scaphoid and lunate are fused to the radius, with motion retained in the mid-carpal joint. This procedure is reserved for selected patients for whom the mid-carpal joint is still maintained. The integrity of the mid-carpal joint requires meticulous medical care to prevent recurrent bouts of synovitis that can destroy this joint.

Total wrist fusion is a predictable operation to achieve a stable wrist and decrease pain. Wrist fusion can be achieved rather successfully either using pins or plates. For the rheumatoid patient, the relatively tenuous soft tissue makes plate fixation a less attractive option.(40) Wound complications can result from over-zealous exposure of the wrist joint for plate fixation. Therefore, the use of Kirschner wires or buried Steinman pins is a much better option for fusion of the rheumatoid wrist. The only problems with imbedding the pins is that migration of the pins can occur, which will require subsequent retrieval. Although wrist fusion in the RA wrist is a predictable operation, some patients are hampered in their activities, particularly when they have bilateral wrist problems. For patients requiring 
treatment for bilateral wrist disease, it has been recommended that the dominant wrist receives wrist arthroplasty whereas the non-dominant wrist is fused in neutral or 10-15 degrees of extension. Recent interest in wrist arthroplasty procedures has spurred new designs of total wrist prosthesis. In contrast to the past designs that are associated with high rate of loosening, implant fracture, peri-prosthetic bone problems, and degenerative wear of the implants, the newer generation of wrist prosthesis appears to have much better outcomes than the prior designs.(41) Given the choice of total wrist fusion and arthroplasty, it is quite difficult for rheumatologists, surgeons and the patients to make an informed choice without full consideration of outcomes and risks associated with these two choices.

A systematic review comparing total wrist fusion (TWF) to total wrist arthroplasty (TWA) revealed that TWF is comparable and possibly preferable to TWA for the rheumatoid wrist. This study demonstrated that existing data do not support the widespread application of TWA as a superior treatment for the RA wrist, especially when taking cost into account.(42) A recent survey of hand surgeons and rheumatologists showed neither group had a definite preference for fusion or arthroplasty as shown by their assigning similar utilities for these two procedures, with TWA being only slightly more preferable. This study indicates that the current interest in TWA for the wrist will require better demonstrated outcomes in order for rheumatologists and surgeons to fully endorse one procedure over the other.(43) Economic analysis shows that, although TWA is more costly due to the cost of the implant material, the incremental cost/quality-adjust-life-year for TWA is much lower than the acceptable range in the national criteria for adoption of a treatment strategy, which is based on the data for renal transplantation.(44)

Reconstruction of the rheumatoid wrist should be the first consideration when the rheumatoid patient presents for hand evaluation, although the opinions of the patient should be taken into account. A painless, stable wrist sets the foundation for future reconstruction of the hand and having a good functional wrist is tantamount for a successfully reconstructed hand.

\section{MCP Joints}

A typical deformity in RA occurs at the MCP joint, which results in volar subluxation of the proximal phalanges and ulnar drift of the fingers.(Figure 4) Chronic synovitis at the MCP joints disrupts the ligamentous support, and the radial stress on the fingers with pinch drives the fingers in the ulnar direction. Although many patients do accommodate to this deformity, the inability to extend the fingers limits the ability to cup the fingers around larger objects, and fine pinch is hampered because the index and middle fingers can no longer oppose the thumb in a tip-to-tip pinch. But what is most distressing to the RA patients is the stigmata of RA caused by the unappealing appearance associated with this deformity. Prior studies have shown that the aesthetic appearance of the MCP joint after reconstruction is just as important for both male and female patients, and patients value the functional and aesthetic gains after reconstruction as measured by the Michigan Hand Outcomes Questionnaire (MHQ).(3)

Fusion of the finger MCP joint is rarely preformed because the arc of motion of the fingers is initiated at the MCP joint. Fusion will position the fingers for stability, but the loss of motion will hinder most of the patient's activities. In the early stages of RA, synovectomy of the MCP joint coupling with a cross intrinsic transfer, in which the ulnar lateral bands are transferred to either the proximal phalanges or the extensor tendons to prevent recurrent ulnar subluxation, is quite effective. Studies have shown that the cross- intrinsic transfer procedure is a good option to restore the posture of the fingers when the joint disease is not too severe and the subluxed fingers can be reduced to the natural posture easily. This 
operation may be considered for patients whose medical management of RA is optimal; otherwise, recurrent bouts of MCP joint synovitis will strain the supporting ligaments and affect outcomes.

For a patient with chronic MCP joint subluxation or with joint destruction, soft tissue reconstruction will not be effective and arthroplasty procedure is needed. Despite the temptation to perform soft tissue reconstruction in a patient with chronic, not easily reduced subluxation at the MCP joints, it is difficult to correct this deformity simply by soft tissue reconstruction alone. MCP joint arthroplasty has the advantage of shortening the skeleton at the MCP joint, which will decrease the tension on the tendons and ligaments contributing to the ulnar deformity and allow the surgeon to position the fingers back onto the hand much more effectively. Studies have shown improved short-term functional and aesthetic outcomes of the silicone metacarpophalangeal joint arthroplasty (SMPA) procedures; $(45$, 46) however, long-term studies have shown a high rate of breakage because of the imperfection of silicone as an arthroplasty material. $(47,48)$ Despite these findings, silicone implants have continued to be popular for the RA hand because of the ease of placement and the relative accessibility for implant replacement. Our current prospective NIH outcomes study on SMPA will measure a variety of objective and patient-rated outcomes parameters in an effort to further define outcomes and complications of this commonly performed RA procedure.(6)

The silicone prosthesis acts as a spacer between the metacarpal and proximal phalanx. A critical sequence in the operation is to centralize the extensor tendon by a combination of imbricating the stretched radial sagittal bands and releasing the tight ulnar sagittal bands and/or the ulnar lateral bands. Although the index and middle fingers can be aligned quite readily, it can be difficult for the ring, and particularly the little finger, to achieve reduction satisfactory. For the little finger, the deforming abductor digiti minimi tendon should be divided in order to make a more effective realignment of the little finger. In some situations, the medullary cavity may be too small to accommodate an implant. Although one may use a bur to open up the medullary cavity, some MCP joints may be too small to accommodate the implant. In these cases, the volar plate arthroplasty procedure, which was described by Tupper(49) before the availability of the silicone implant, may be applied. The proximal volar plate is detached and sutured to the dorsum of the metacarpal in order to provide a buffer between the bone ends. (Figure 5) The problem with this operation is that joint stability cannot be achieved predictably and recurrent deviation is likely. Nevertheless, when an implant arthroplasty cannot be performed, this operation can be used to achieve a pseudoarthrosis between the bone ends. The standard protocol after SMPA is to initiate a six-week dynamic extension exercise protocol. However, recent evidence has shown that a static protocol in which the patient is splinted for 6 weeks may be equally as effective and less troublesome for the patient.(50) More studies should be performed to see if the static protocol is equivalent to the dynamic protocol because the direct and indirect cost savings from employing a simpler therapy protocol is substantial.

The future of arthroplasty for the RA hand requires the design of an easily inserted prosthesis for the MCP joint that has sufficient longevity for the remaining lifetime of the rheumatoid patient. The current silicone implant, although easy to insert, is not an anatomic design and is prone to fractures. Despite fracture of the silicone implant, scar tissues do form around the implants, creating sufficient support that implant replacement, in most cases, is not necessary. An increasing interest exists to use the unconstrained, two-piece pyrocarbon implant for the rheumatoid MCP joint. Although this implant is well suited for the osteoarthritic MCP joints, it is not particularly applicable for the RA condition because ligament laxity and deforming forces makes recurrent joint subluxation likely.(51)) 


\section{Tendon rupture}

Tendon rupture is a common problem in patients with RA. (Figure 6) There are two reasons for tendon rupture: (1) abrasion of the tendon over bony prominence, such as the eroded distal ulna or the distal pole of the scaphoid (rupturing the flexor pollicis longus tendon), or (2) weakening of the tendon by synovial invasion. When the distal ulna is eroded and subluxed dorsally, the little finger extensor tendon is often the first to rupture when it crosses over the head of the ulna. The tendons may already be weakened by synovial hypertrophy within the extensor compartment, and are prone to rupture when encountering mechanical trauma. Once the little finger extensor tendon is ruptured, the ring tendon will move over ulnarly and will rupture eventually. Referral to hand surgeons may be delayed because RA patients are quite adaptable to their deformities. The intact juncturae between the ring and little finger may still extend the little finger, albeit incompletely. An astute rheumatologist may detect the tendon rupture when the little finger cannot extend while the other fingers are flexed (by checking the common extensor tendons) due to rupture of the extensor digiti quinti minimi. The definitive treatment for the tendon rupture is addressing the offending causes by removing the head of the ulna and excising the hypertrophic synovial tissue over the extensor mechanism.

Strategies for extensor tendon reconstruction follow the principles for tendon transfer.(52) When the little finger extensor tendon is ruptured, the distal end of the intact tendon can be transferred end-to-side to the intact ring finger. When the ring and little finger extensor tendons are ruptured, the ideal transfer is to use the extensor indicis proprius (EIP) tendon to power the ring and little fingers. It is not advisable to suture the extensor tendons of the ring and little finger to the intact middle finger extensor tendon. Although extension can be achieved in this form of tendon transfer, the acute angle from the little finger to the middle finger extensor tendon causes an unacceptable abduction of the little finger. When the middle, ring, and little fingers are ruptured, it raises the concern whether the RA patient has received the appropriate attention. In this situation, the EIP is transferred to power the ring and little fingers and the middle finger extensor tendon can be transferred to the index communis extensor tendon. Because all four fingers will be powered by the extensor tendons to the index finger, the strength of extension will be diminished to a great extent. An alternative strategy is to transfer the FDS tendon from either the middle or the ring finger to power the ring and little fingers, while the EIP tendon is used to power the middle finger. In the highly unusual situation when all four extensor tendons are ruptured, the tendon transfer option requires the use of both the FDS tendons from the middle and ring fingers to power the index/middle and ring/little fingers, respectively. Because using the FDS for finger extension is not a synergistic transfer, learning this tendon transfer can be difficult for some patients. However, the FDS has over $70 \mathrm{~mm}$ of excursion and most patients can adapt due to the long excursion of this "smart" tendon. The FDS is recommended to pass either through the ulnar or radial subcutaneous tunnels to avoid potential scarring from tunneling through the interosseous membrane. There should be sufficient amount of tendon length from the FDS tendons that is transected at the A1 pulley level to reach the ruptured tendons over the dorsal wrist crease. Occasionally, the EPL tendon may be ruptured by synovial invasion. The EIP tendon transfer is ideal for reconstructing the ruptured EPL tendon.

Other than tendon ruptures causing an inability to extend the fingers, there are three additional reasons why the fingers cannot extend at the MCP joint. First, the extensor tendon is subluxed in the valley between the heads of the metacarpals. The extensor tendons now lie below the axes of the MCP joints and instead will serve as flexors rather than extensors. This situation is common and can be identified readily by observing the tendons lying ulnar to the heads of the metacarpals. When the fingers are extended passively, the extensor tendons may relocate over the heads of the metacarpals and partially extend the fingers. 
Second, the MCP joints may dislocate, resulting in contracture of the joint and inability to extend the finger. This will be apparent by radiographs that demonstrate this deformity. Third, the posterior interosseous nerve (PIN) is compressed by the swelling at the elbow joint. This is a very uncommon condition, and I have never observed this finding,

\section{Finger Deformities: Boutonniere versus Swan-Neck}

The joints in the hand and wrist are linked, and the deformity in a proximal joint will affect the position of the joints distally. This situation is analogous to a train derailment, when collapse of one segment of the train cart results in a zigzag deformity distally.(53) For the RA hand, consideration of reconstruction for swan-neck and boutonniere's deformity is quite different. Swan-neck deformity usually causes a functional problem because the patient is unable to make a full fist due to the hyper-extended posture of the PIP joint, (Figure 7) whereas in boutonnière's deformity, particularly in the ulnar digits, the concern is more of an aesthetic than a functional concern. In this deformity, the patient is still able to make a fist, but the flexed-posture of the PIP joint usual drives the patient to seek care. It has been stated by Dr Adrian Flatt that correction of boutonniere's deformity caused by trauma is difficult, and the correction of boutonniere's deformity caused by rheumatoid diseases is virtually impossible. The reason for this pessimism is due to the potential risk in changing the flexed posture of the PIP joint into an extended posture, which may be functionally limiting, although aesthetically more pleasing. Therefore, it is important to understand why RA patients seek care for their hand problems because some patients may have purely aesthetic concerns, without true functional issues. It has been found that aesthetic consideration is a critical determinant of patient satisfaction in RA, $(3,54)$ but the classic teaching in RA hand surgery is that the presence of deformity is not an indication for surgical reconstruction.(55)

In contrast to the multiple etiologies of swan-neck deformity, the etiology of boutonnière's deformity is a problem at the PIP joint. (Figure 8) Synovitis in the PIP joint causes an elongation of the central slip. The lateral bands sublux below the axis of rotation resulting in shortening of the retinacular ligaments. These constellation of events result in an incomplete extension of the PIP joint and hyperextension of the DIP joint due to tightening of the lateral bands. When the surgeon evaluates these RA finger deformities for surgical treatment, he/ she needs to classify the deformity into flexible or fixed deformity. For flexible deformity, the treatment is soft tissue reconstruction, and for fixed deformity, the treatment is joint fusion or implant arthroplasty. For flexible chronic boutonniere's deformity, the treatment is joint synovectomy, tightening of the stretched central tendon and bringing the lateral bands dorsally. However, one of the difficulties with this operation is the unpredictability of the correction. Therefore, it is more preferable to prevent chronic PIP joint synovitis that can contribute to this deformity. If synovitis at the PIP joint persists for over three months despite maximal medical management, synovectomy should be performed to avoid stretching of the support ligaments and the central tendon and to prevent articular destruction by the invasive synovial tissue. When the articular surface is destroyed, the options are implant arthroplasty or fusion. There is a greater interest to perform implant arthroplasty for the PIP joint. However, implant arthroplasty must be considered carefully for boutonniere's deformity because the need for excessive excision of the PIP joint to insert an implant will require removal of the collateral ligaments, which will destabilize the joint. It is even more problematic in RA patients when one uses an unconstrained implant such as the pyrocarbon implant because the support ligaments are already laxed.

For swan-neck deformities, the treatment will be determined by where the problem originates because this deformity can arise from the DIP joint, the PIP joint, or the MCP joint. For example, the mallet posture from DIP joint disease may cause the cascade of 
zigzag deformities proximally to result in the swan-neck deformity. The treatment should be focused at the DIP joint by fusion. On the other hand, PIP joint disease may stretch out the volar plate preferentially or even rupture of the FDS tendon to cause a hyperextension posture of the PIP joint. At the MCP joint level, subluxation of the joint and extensor tendon mechanism can result in ulnar intrinsic tendon tightness leading to this deformity. Therefore, when considering correction of swan-neck deformities for the RA patient, the physician must understand where the pathology starts so that the appropriate joint can be treated. Again, classification of the swan-neck deformity can be divided into either flexible or fixed deformity. For example, in a patient with flexible deformity, one can entertain soft tissue reconstruction by releasing the tight ulnar intrinsic tendon, restraining the PIP joint by volar plate advancement, or by the FDS tenodesis procedure, which is a simpler and preferable operation by dividing a proximal slip to the FDS tendon and suturing it to the A1 pulley. The PIP joint is set at a 30 degree flexion posture to prevent hyperextension. If the lateral bands are dorsal-riding, the interval between the lateral bands and the central tendon can be incised and the joint passively flexed to bring the lateral bands volarly. A curve linear incision is made over the dorsal PIP joint so upon closure, the recruitment of lateral skin can close the incision with the PIP joint flexed partially. Another option is the Littler oblique retinacular ligament reconstruction,(56) which not only releases the extension contracture of the PIP joint, but can also flex this joint. In this operation, the ulnar lateral band is detached proximally and the tendon is passed volar to the axis of the PIP joint under the Cleland's ligament to simultaneously extend the DIP joint and flex the PIP joint. The tendon is then sutured into the bone at the proximal phalanx to set the finger posture to a more natural flexed PIP joint posture. This operation elegantly treats the flexed posture of the DIP joint by pulling on the lateral band. If the deformity is fixed, then fusion or implant arthroplasty is the only option. However, fusion of the PIP joint must be considered carefully after understanding the patients' needs in order to avoid causing other functional issues. In some cases, doing nothing may be a better option.

\section{Thumb deformities}

It is often said that the thumb constitutes 50\% of the hand function. Therefore, the most gratifying part of rheumatoid hand surgery is to reconstruct the thumb so that the patient has a stable, mobile thumb to improve grip strength, posture, and appearance. The thumb can also be classified into a boutonniere posture in which MCP joint is flexed and the IP joint is hyperextended, or the less common swan-neck deformity in which the MCP joint is extended and the IP joint is flexed. (Figure 9) For boutonniere thumb deformity, the MCP joint is the problem and fusion of the MCP joint will shorten the thumb a little bit, which will balance the extensor mechanism to give the thumb a more natural posture. For the swan-neck thumb, the problem is at the carpometacarpal (CMC) joint, for which one can either fuse the $\mathrm{CMC}$ joint or perform an arthroplasty procedure with either a tendon or an implant. Many RA patients do present with instability of both the thumb MCP and the IP joints. If the thumb CMC joint is mobile, simultaneous fusion of the MCP and the IP joint can be entertained; however, this will markedly limit the movement of the thumb due to fusion of the thumb at two different levels. In certain situations, one should consider fusion of the thumb MCP joint as the primary operation because instability of the thumb at this level is most distressing as the joint subluxation can affect power grip. During exposure for the fusion operations, the extensor mechanisms can be lengthened or shortened depending on the posture of the reconstructed thumb.

\section{Summary}

RA hand surgery can markedly improve the patients' quality of life. However, it cannot be a cookbook approach in treating the patient. An experienced RA hand surgeon will listen 
carefully to the patient and consider his/her needs. Collaboration with the referring rheumatologists who have an intimate knowledge of the patients' overall disease, psychosocial issues, and functional requirements will be helpful in deciding whether an operation is needed and what type of procedures should be performed. The patients and the referral rheumatologists need to understand that referral for hand surgery consultation does not necessarily equate to having a surgical plan. Careful consideration of the patient's needs and desired outcomes for the surgical treatment will play into whether surgical procedures are necessary. To create trust by all parties, the initial surgical treatment must yield predictable outcomes, for example, thumb MCP joint fusion and extensor tendon reconstructions can be the initial consideration. It is also helpful to outline for the patient a sequence of treatment priorities in order to plan for a long-term relationship. Informing the rheumatologists of the plans will help in their management of the patients before and after surgery. Although the incidence of rheumatoid hand surgery appears to be decreasing, there is still a need for hand surgeons to be involved in the care of the rheumatoid hands because a substantial number of patients may not be responsive to medications and will still develop hand deformities. It is also uncertain whether these new medications simply delay the inevitable progression of hand and wrist diseases. Hand surgery should remain a partner with rheumatology in the coordinated care of patients whose hand diseases may be treated by the continued innovations in arthroplasty procedures. Ultimately, high impact clinical studies must be conducted by hand surgeons to support the surgical approaches and guide the appropriate management of the RA population.

\section{Acknowledgments}

Supported in part by a grant from the National Institute of Arthritis and Musculoskeletal and Skin Diseases (R01 AR047328) and a Midcareer Investigator Award in Patient-Oriented Research (K24 AR053120) (to Dr. Kevin C. Chung).

\section{References}

1. Kotsis SV, Chung KC. A qualitative assessment of rheumatoid hand surgery in various regions of the world. J Hand Surg. 2005; 30A:649-657.

2. Alderman AK, Chung KC, Kim HM, et al. Effectiveness of rheumatoid hand surgery: contrasting perceptions of hand surgeons and rheumatologists. J Hand Surg. 2003; 28A:3-11.

3. Alderman AK, Arora AS, Kuhn L, et al. An analysis of women's and men's surgical priorities and willingness to have rheumatoid hand surgery. J Hand Surg. 2006; 31A:1447-1453.

4. Alderman AK, Ubel PA, Kim HM, et al. Surgical management of the rheumatoid hand: consensus and controversy among rheumatologists and hand surgeons. J Rheumatol. 2003; 30:1464-1472. [PubMed: 12858442]

5. Michaud K, Messer J, Choi HK, et al. Direct medical costs and their predictors in patients with rheumatoid arthritis: a three-year study of 7,527 patients. Arthritis Rheum. 2003; 48:2750-2762. [PubMed: 14558079]

6. Chung KC, Burns PB, Wilgis EF, et al. A multi-center clinical trial in rheumatoid arthritis patients by comparing silicone metacarpophalangeal joint arthroplasty with medical treatment. JAMA. submitted.

7. Lawrence RC, Helmick CG, Arnett FC, et al. Estimates of the prevalence of arthritis and selected musculoskeletal disorders in the United States. Arthritis Rheum. 1998; 41:778-799. [PubMed: 9588729]

8. De la Mata Llord J, Palacios Carvajal J. Rheumatoid arthritis: are outcomes better with medical or surgical management? Orthop. 1998; 21:1085-1086.

9. Alderman AK, Chung KC, DeMonner S, et al. Large area variations in the surgical management of the rheumatoid hand. Surg Forum. 2001; LII:479-481.

10. Alderman AK, Chung KC, Demonner S, et al. The rheumatoid hand: a predictable disease with unpredictable surgical practice patterns. Arthritis Rheum. 2002; 47:537-542. [PubMed: 12382304] 
11. Ghattas L, Mascella F, Pomponio G. Hand surgery in rheumatoid arthritis: state of the art and suggestions for research. Rheumatology. 2005; 44:834-845. [PubMed: 15797979]

12. Klockars M, Koskela RS, Jarvinen E, et al. Silica exposure and rheumatoid arthritis: a follow up study of granite workers 1940-81. Br Med J (Clin Res Ed). 1987; 294:997-1000.

13. Steenland K, Goldsmith DF. Silica exposure and autoimmune diseases. Am J Ind Med. 1995; 28:603-608. [PubMed: 8561170]

14. Klareskog L, Stolt P, Lundberg K, et al. A new model for an etiology of rheumatoid arthritis: smoking may trigger HLA-DR (shared epitope)-restricted immune reactions to autoantigens modified by citrullination. Arthritis Rheum. 2006; 54:38-46. [PubMed: 16385494]

15. Cornelis F, Faure S, Martinez M, et al. New susceptibility locus for rheumatoid arthritis suggested by a genome-wide linkage study. Proc Natl Acad Sci U S A. 1998; 95:10746-10750. [PubMed: 9724775]

16. Aho K, Koskenvuo M, Tuominen J, et al. Occurrence of rheumatoid arthritis in a nationwide series of twins. J Rheumatol. 1986; 13:899-902. [PubMed: 3820198]

17. Silman AJ, MacGregor AJ, Thomson W, et al. Twin concordance rates for rheumatoid arthritis: results from a nationwide study. Br J Rheumatol. 1993; 32:903-907. [PubMed: 8402000]

18. Begovich AB, Carlton VE, Honigberg LA, et al. A missense single-nucleotide polymorphism in a gene encoding a protein tyrosine phosphatase (PTPN22) is associated with rheumatoid arthritis. Am J Hum Genet. 2004; 75:330-337. [PubMed: 15208781]

19. Lee AT, Li W, Liew A, et al. The PTPN22 R620W polymorphism associates with RF positive rheumatoid arthritis in a dose-dependent manner but not with HLA-SE status. Genes Immun. 2005; 6:129-133. [PubMed: 15674368]

20. Stastny P, Fink CW. HLA-Dw4 in adult and juvenile rheumatoid arthritis. Transplant Proc. 1977; 9:1863-1866. [PubMed: 146287]

21. Gregersen PK, Silver J, Winchester RJ. The shared epitope hypothesis. An approach to understanding the molecular genetics of susceptibility to rheumatoid arthritis. Arthritis Rheum. 1987; 30:1205-1213. [PubMed: 2446635]

22. Winchester R. The molecular basis of susceptibility to rheumatoid arthritis. Adv Immunol. 1994; 56:389-466. [PubMed: 7521116]

23. Deighton CM, Walker DJ, Griffiths ID, et al. The contribution of HLA to rheumatoid arthritis. Clin Genet. 1989; 36:178-182. [PubMed: 2676268]

24. Schett G, Stach C, Zwerina J, et al. How antirheumatic drugs protect joints from damage in rheumatoid arthritis. Arthritis Rheum. 2008; 58:2936-2948. [PubMed: 18821703]

25. Matsumoto, AK.; Bathon, JM.; Bingham, CO. Rheumatoid Arthritis Treatment. The John Hopkins Arthritis Center; http://www.hopkins-arthritis.org/arthritis-info/rheumatoid-arthritis/rheum_treat.html

26. Fardet L, Kassar A, Cabane J, et al. Corticosteroid-induced adverse events in adults: frequency, screening and prevention. Drug Saf. 2007; 30:861-881. [PubMed: 17867724]

27. Gibofsky A. Combination therapy for rheumatoid arthritis in the era of biologicals. HSS J. 2006; 2:30-41. [PubMed: 18751844]

28. Lard LR, Visser H, Speyer I, et al. Early versus delayed treatment in patients with recent-onset rheumatoid arthritis: comparison of two cohorts who received different treatment strategies. Am J Med. 2001; 111:446-451. [PubMed: 11690569]

29. Chen YF, Jobanputra P, Barton P, et al. A systematic review of the effectiveness of adalimumab, etanercept and infliximab for the treatment of rheumatoid arthritis in adults and an economic evaluation of their cost-effectiveness. Health Technol Assess. 2006; 10:iii-iv. xi-xiii, 1-229. [PubMed: 17049139]

30. Nurmohamed MT, Dijkmans BA. Are biologics more effective than classical disease-modifying antirheumatic drugs? Arthritis Res Ther. 2008; 10:118. [PubMed: 18828888]

31. Weinblatt ME. Rheumatoid arthritis: more aggressive approach improves outlook. Cleve Clin J Med. 2004; 71:409-413. [PubMed: 15195775]

32. Furst DE, Schiff MH, Fleischmann RM, et al. Adalimumab, a fully human anti tumor necrosis factor-alpha monoclonal antibody, and concomitant standard antirheumatic therapy for the 
treatment of rheumatoid arthritis: results of STAR (Safety Trial of Adalimumab in Rheumatoid Arthritis). J Rheumatol. 2003; 30:2563-2571. [PubMed: 14719195]

33. St Clair EW, van der Heijde DM, Smolen JS, et al. Combination of infliximab and methotrexate therapy for early rheumatoid arthritis: a randomized, controlled trial. Arthritis Rheum. 2004; 50:3432-3443. [PubMed: 15529377]

34. Maini RN, Breedveld FC, Kalden JR, et al. Sustained improvement over two years in physical function, structural damage, and signs and symptoms among patients with rheumatoid arthritis treated with infliximab and methotrexate. Arthritis Rheum. 2004; 50:1051-1065. [PubMed: 15077287]

35. Taleisnik, J.; Ruby, LK. Arthritis Deformity: Resection Arthroplasty and Fusion. In: Cooney, WP.; Linscheid, RL.; Dobyns, JH., editors. The Wrist. Vol. 2. St. Louis: Mosby; 1998.

36. Chamay A, Della Santa D. Radiolunate arthrodesis in rheumatoid wrist (21 cases). Ann Chir Main Memb Super. 1991; 10:197-206. [PubMed: 1718354]

37. Borisch N, Haussmann P. Radiolunate arthrodesis in the rheumatoid wrist: a retrospective clinical and radiological longterm follow-up. J Hand Surg [Br]. 2002; 27:61-72.

38. Lau FH, Chung KC. William Darrach, MD: his life and his contribution to hand surgery. J Hand Surg. 2006; 31A:1056-1060.

39. Fujita S, Masada K, Takeuchi E, et al. Modified Sauve-Kapandji procedure for disorders of the distal radioulnar joint in patients with rheumatoid arthritis. Surgical technique. J Bone Joint Surg Am. 2006; 88(Suppl 1 Pt 1):24-28. [PubMed: 16510797]

40. Nalebuff EA. Rheumatoid hand surgery - update. J Hand Surg. 1983; 8A:678-682.

41. Radmer S, Andresen R, Sparmann M. Wrist arthroplasty with a new generation of prostheses in patients with rheumatoid arthritis. J Hand Surg [Am]. 1999; 24:935-943.

42. Cavaliere CM, Chung KC. A systematic review of total wrist arthroplasty compared with total wrist arthrodesis for rheumatoid arthritis. Plast Reconstr Surg. 2008; 122:813-825. [PubMed: 18766045]

43. Cavaliere C, Chung KC. A decision analysis of arthrodesis versus arthroplasty for the rheumatoid wrist. J Hand Surg. In Press.

44. Cavaliere C, Chung KC. A cost-utility analysis comparing total wrist fusion and total wrist arthroplasty for the rheumatoid wrist. Journal of Bone and Joint Surgery. Submitted.

45. Chung KC, Kotsis SV, Kim HM. A prospective outcomes study of Swanson metacarpophalangeal joint arthroplasty for the rheumatoid hand. J Hand Surg. 2004; 29A:646-653.

46. Chung KC, Kowalski CP, Kim HM, et al. Patient outcomes following Swanson silastic metacarpophalangeal joint arthroplasty in the rheumatoid hand: a systematic overview. J Rheumatol. 2000; 27:1395-1402. [PubMed: 10852260]

47. Bass RL, Stern PJ, Nairus JG. High implant fracture incidence with Sutter silicone metacarpophalangeal joint arthroplasty. J Hand Surg [Am]. 1996; 21:813-818.

48. Burgess SD, Kono M, Stern PJ. Results of revision metacarpophalangeal joint surgery in rheumatoid patients following previous silicone arthroplasty. J Hand Surg [Am]. 2007; 32:15061512.

49. Tupper JW. The metacarpophalangeal volar plate arthroplasty. J Hand Surg [Am]. 1989; 14:371375.

50. Ring D, Simmons BP, Hayes M. Continuous passive motion following metacarpophalangeal joint arthroplasty. J Hand Surg [Am]. 1998; 23:505-511.

51. Chung KC, Ram AN, Shauver M. Outcomes of pyrolytic carbon arthroplasty for the proximal interphalangeal joint. Plast Reconstr Surg. In Press.

52. Chung, K. Operative Techniques: Hand and Wrist Surgery. 1. Philadelphia: Saunders/Elsevier; 2007. Tendon transfers for the rheumatoid arthritis patient; p. 757-770.

53. Stack HG, Vaughan-Jackson OJ. The zig-zag deformity in the rheumatoid hand. Hand. 1971; 3:6267. [PubMed: 5098339]

54. Chung KC, Kotsis SV, Kim HM, et al. Reasons why rheumatoid arthritis patients seek surgical treatment for hand deformities. J Hand Surg. 2006; 31A:289-294.

55. Souter WA. Planning treatment of the rheumatoid hand. Hand. 1979; 11:3-16. [PubMed: 488774] 
56. Thompson JS, Littler JW, Upton J. The spiral oblique retinacular ligament (SORL). J Hand Surg [Am]. 1978; 3:482-487. 


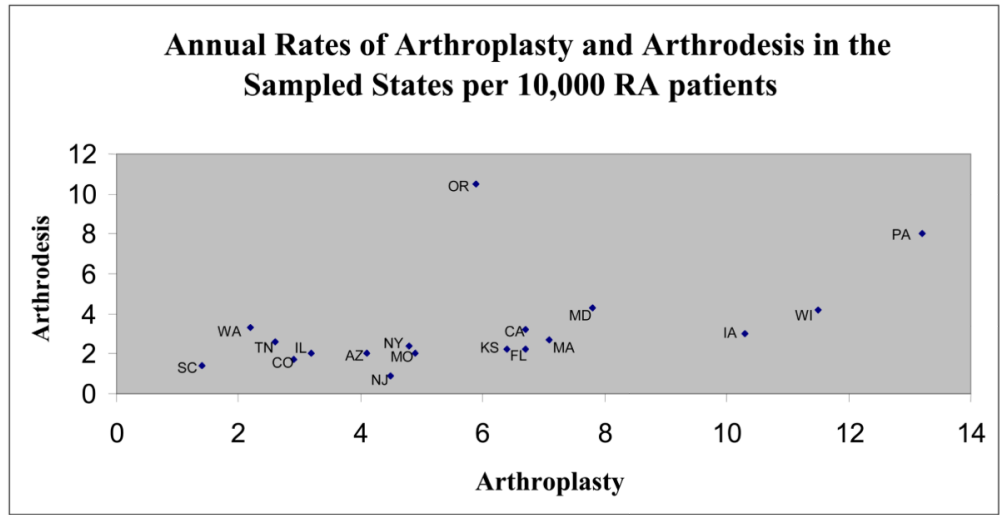

Figure 1.

Annual Rates of Arthroplasty and Arthodesis in a Sample of States per 10,000 RA patients. (Source: Alderman, A. K et al. The rheumatoid hand: a predictable disease with unpredictable surgical practice patterns. Arthritis Rheum 47: 537-542, 2002.) 


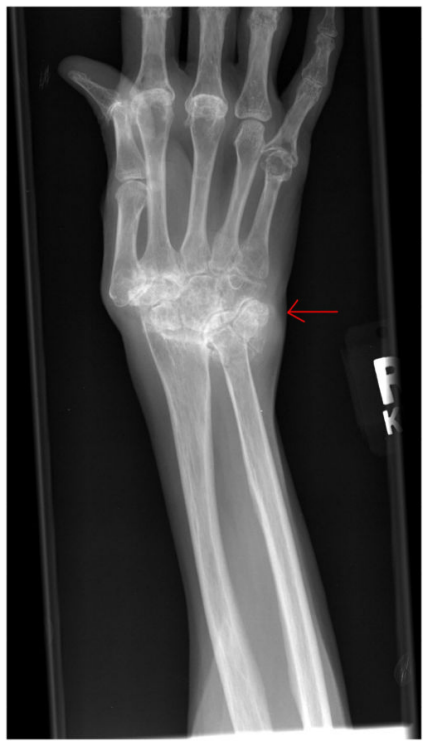

Figure 2.

Radiograph of typical caput ulna appearance in an RA patient. Please note the impaction of the distal ulna on the carpus. (Black Arrow) 


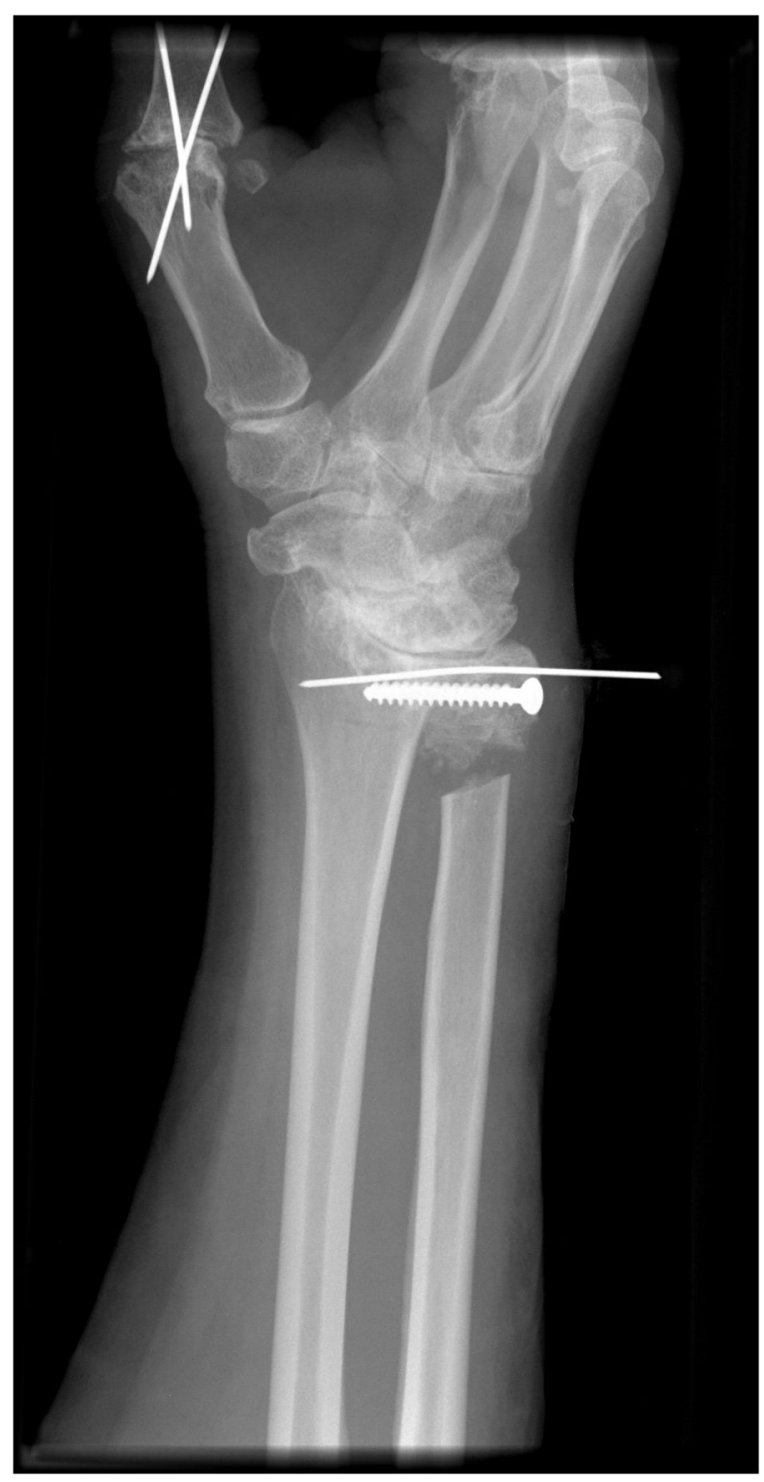

Figure 3.

Post-operative wrist radiograph of a 48-year-old female treated with the Masada Shelf Arthoplasty. 


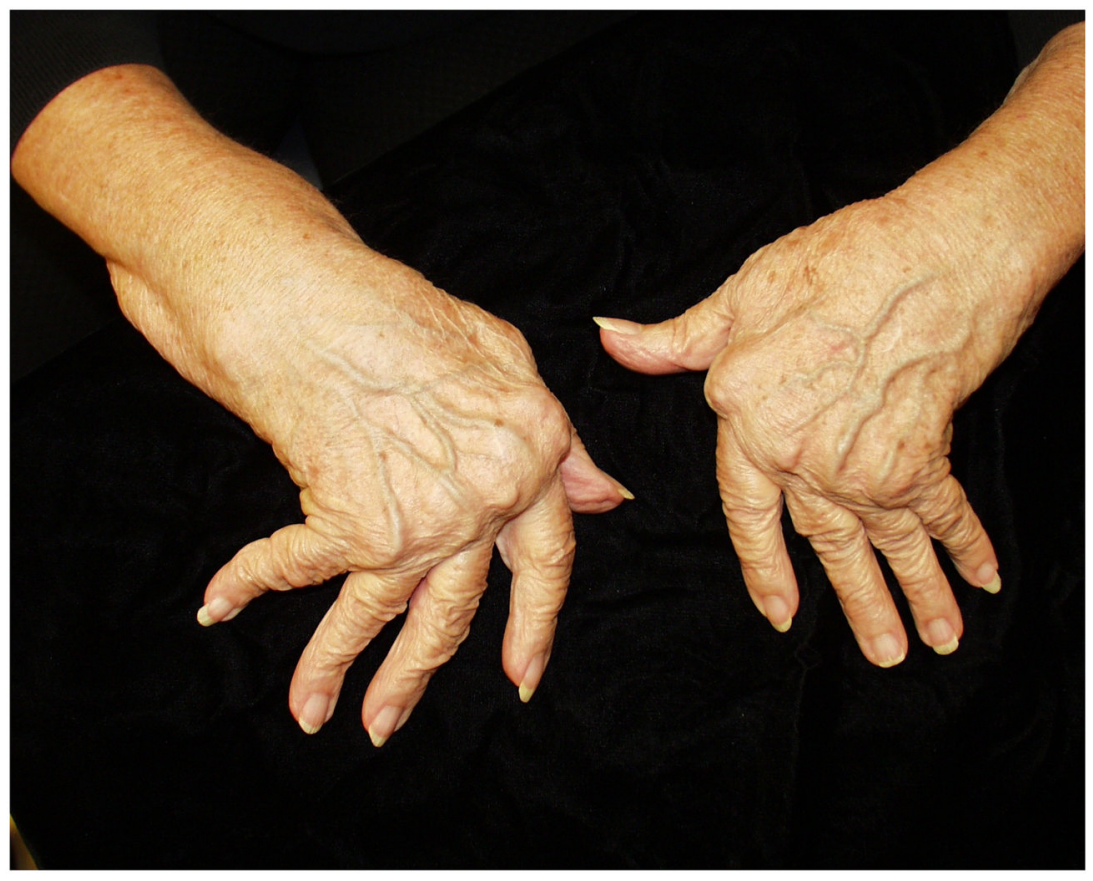

Figure 4.

Ulnar deviation caused by an imbalance in tendon forces. 


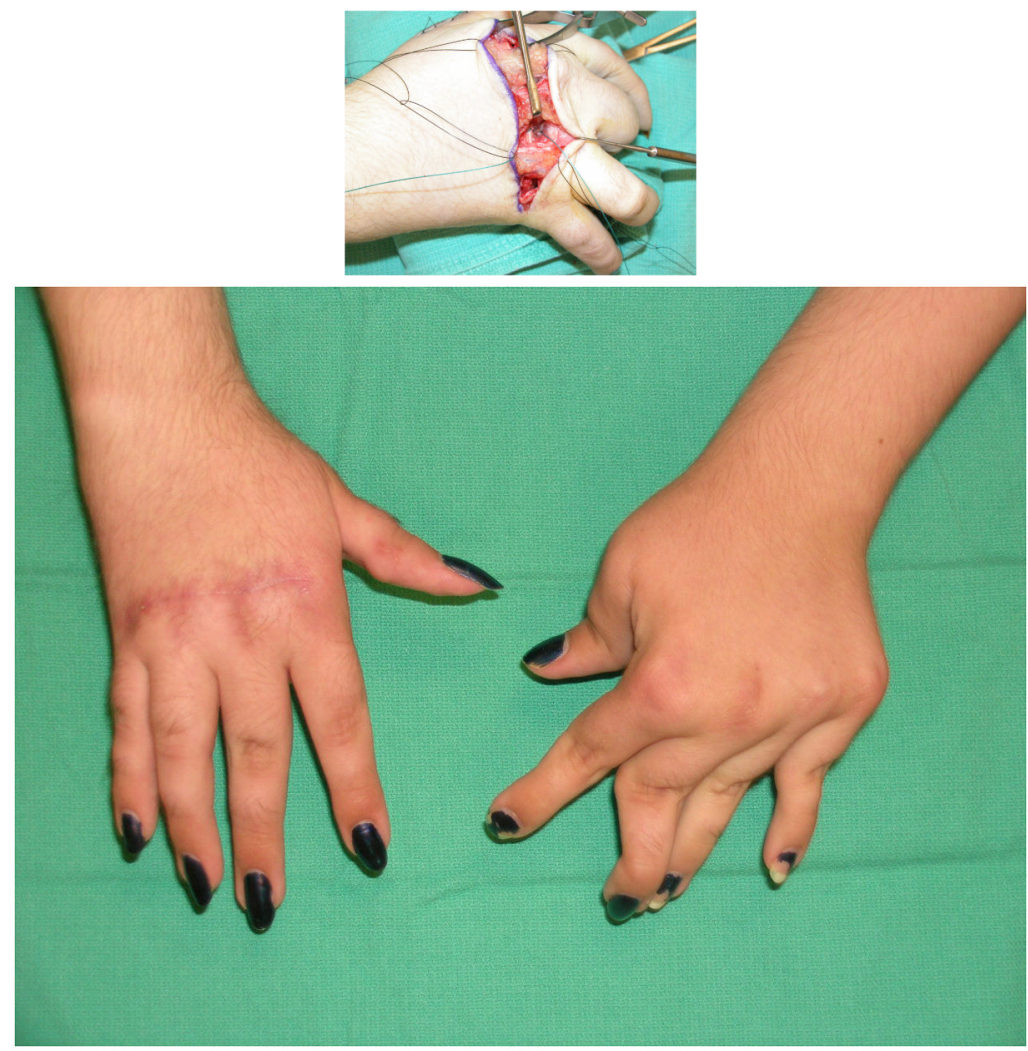

Figure 5.

A. Intra-operative view of a 23-year-old woman who underwent the Tupper procedure on her right hand. The volar plate is detached proximally and sutured to the soft tissue on the dorsum of the resected head of the metacarpal. B. Postoperative view. Please note the differences in appearance between her right and left hand. 


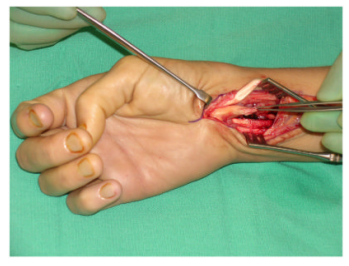

Figure 6.

Right index finger flexor tendon rupture caused by synovial hypertrophy over the wrist. 


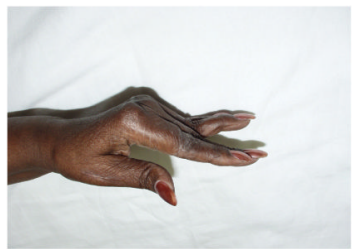

Figure 7.

Swan-neck deformity with hyperextended PIP joint. 


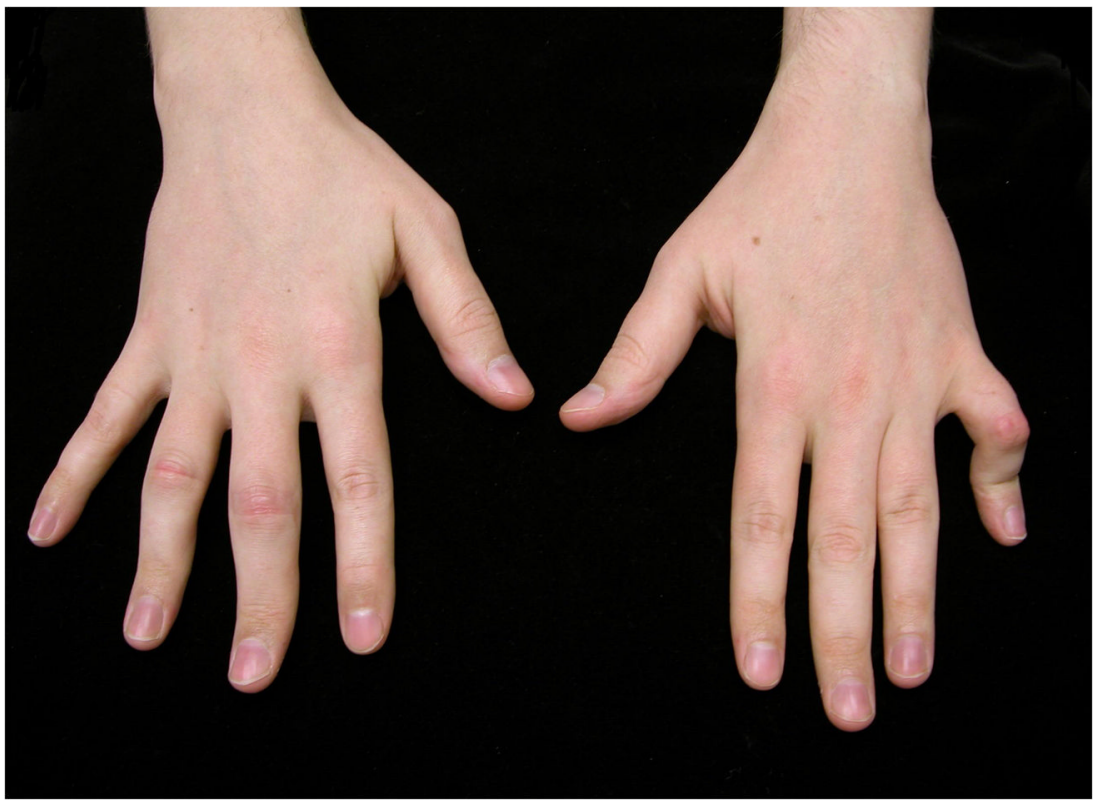

Figure 8.

Boutonniere's Deformity of the little finger. Because the patient can still make a full fist, correction of this deformity is not advisable for the risk of causing a more functionally limiting PIP extension posture. 


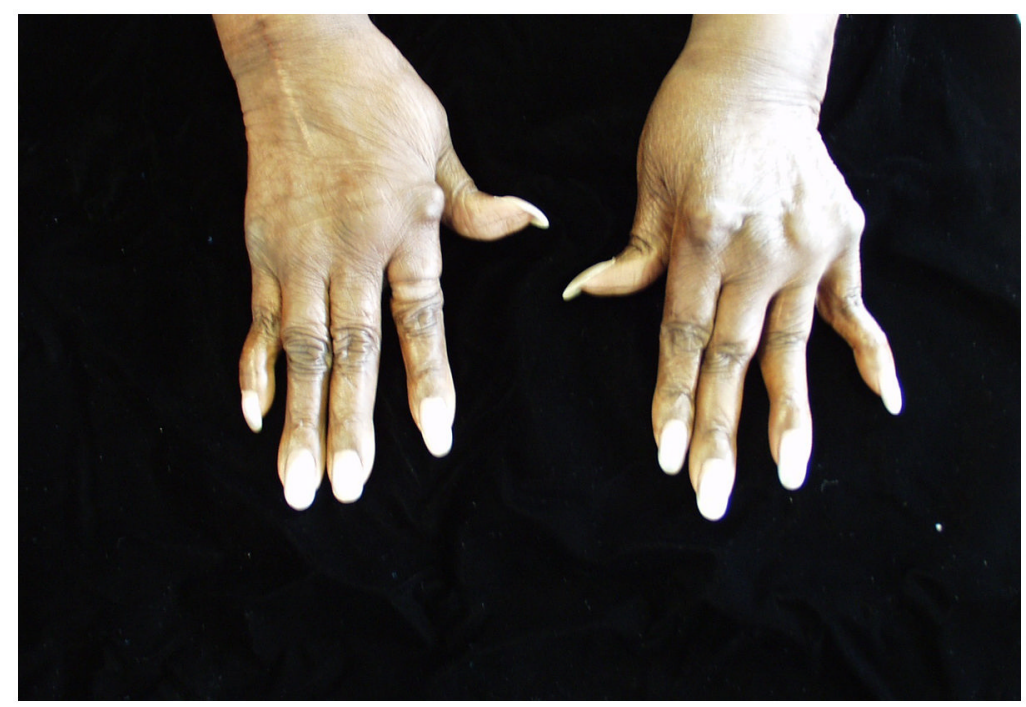

Figure 9.

Swan-neck deformity of the thumb caused by CMC joint disease. 